\title{
Endoscopic ultrasound-guided radiofrequency ablation of unresectable pancreatic cancer with low ablation power and multiple applications: a preliminary study of 11 patients
}

\author{
Jinlin Wang ${ }^{1,2 \#}$, Yun Wang ${ }^{2 \#}$, Yuchong Zhao ${ }^{2}$, Xiaoli Wu ${ }^{2}$, Min Zhang ${ }^{2}$, Wei Hou ${ }^{2}$, Qian Chen $^{2}$, \\ Bin Cheng ${ }^{2}$
}

${ }^{1}$ Department of Oncology, Tongji Hospital, Tongji Medical College, Huazhong University of Science and Technology, Wuhan, China; ${ }^{2}$ Department of Gastroenterology and Hepatology, Tongji Hospital, Tongji Medical College, Huazhong University of Science and Technology, Wuhan, China

Contributions: (I) Conception and design: J Wang, Y Wang, Q Chen, B Cheng; (II) Administrative support: Q Chen, B Cheng; (III) Provision of study materials or patients: J Wang, Y Wang, Q Chen; (IV) Collection and assembly of data: J Wang, Y Wang, M Zhang, W Hou; (V) Data analysis and interpretation: J Wang, Y Wang, X Wu, Q Chen, B Cheng; (VI) Manuscript writing: All authors; (VII) Final approval of manuscript: All authors.

\#These authors contributed equally to this work.

Correspondence to: Bin Cheng. Department of Gastroenterology and Hepatology, Tongji Hospital, Tongji Medical College, Huazhong University of Science and Technology, Jiefang Avenue 1095, Wuhan 430030, China. Email: b.cheng@tjh.tjmu.edu.cn. Qian Chen. Department of Gastroenterology and Hepatology, Tongji Hospital, Tongji Medical College, Huazhong University of Science and Technology, Jiefang Avenue 1095, Wuhan 430030, China. Email: chenqian201579@yahoo.com.

Backgroundk Endoscopic ultrasound (EUS)-guided radiofrequency ablation (RFA) is a novel modality in the treatment of solid tumours. The aim of the study is to evaluate the technical feasibility, safety and efficacy of multiple-round EUS-RFA with low ablation power for unresectable pancreatic cancer.

Methods: We conducted a retrospective analysis of eleven cases with unresectable pancreatic cancer who underwent EUS-RFA between November 2013 and November 2018. For each lesion, RITA 1500X radiofrequency generator was used to deliver 5-10 watts ablation power for 90 seconds, repeatedly. Eight cases underwent the same procedure one week later. Additionally, one patient with the lesion size of $29.7 \mathrm{~mm}$ underwent 8 total sessions of RFA every other week.

Results: The procedure was successful in all cases and no major adverse events were observed. The post procedure imaging studies and serum CA19-9 level were performed 1 month after procedure, showing two patients had decreased lesion sizes and five patients had decreased serum CA19-9 level. Follow-up duration ranged 2 to 12 months. The patient who underwent 8 total sessions of RFA survived 12 months after followup and showed increased tumour apparent diffusion coefficient (ADC) value and $20 \%$ ablated area inside the tumour.

Conclusions: A multiple-round ablation with optimal RFA energy could be a technically feasible, safe and short-term efficacy option for those patients with unresectable pancreatic cancer.

Keywords: Pancreatic cancer; adjuvant therapy; endoscopic ultrasound-guided radiofrequency ablation (EUS-RFA)

Submitted Feb 28, 2020. Accepted for publication Sep 18, 2020.

doi: 10.21037/apm-20-1468

View this article at: http://dx.doi.org/10.21037/apm-20-1468 


\section{Introduction}

Pancreatic cancer often has a poor prognosis, with a 5-year survival rate below $10 \%$ and a median survival of less than 6 months (1). Conventional treatment approaches, such as surgery, radiation, chemotherapy, or combinations of these, have limited benefit for this aggressive cancer (2). Although several recent randomized controlled phase III trials have suggested potential approaches to improve pancreatic cancer treatment, resulting in median survivals of 8 to 13 months $(3,4)$, better treatment options are required for patients with advanced pancreatic cancer.

Radiofrequency ablation (RFA) is a well-established technique that produces a high level of heat within the tumour, leading to necrosis, followed by replacement of the dead tumour cells with scar tissue. It works well in treating various types of cancer such as liver, lung, and kidney cancers, causing few side effects without worsening quality of life in these patients $(5,6)$. During laparotomy, RFA has been introduced to serve as a palliative procedure in inoperable pancreatic cancers $(7,8)$. Nevertheless, ablating part of the pancreas might prove rather challenging because of its complex anatomy and the difficulty to precisely localize the target. Additionally, pancreatic tissue, which is biologically highly thermosensitive, when subjected to high levels of heat will cause an inflammatory response, followed by oedema, fibrotic changes, and finally, cystic transformation $(7,8)$.

With the development of linear-array endoscopic ultrasound (EUS), RFA with EUS guidance (EUS-RFA) allows for real-time imaging deep in the pancreas, which would avoid possible injury to the adjacent tissues (9). Promising results has been reported for EUS-RFA of pancreatic cancer as a technically feasible and minimally invasive option and a novel future modality in a porcine model (10). However, there is no standardized protocol for pancreatic RFA since the optimal thermal kinetic characteristics for the pancreas have not been determined. For larger pancreas masses, it is still difficult to safely induce complete coagulation necrosis. The currently achievable coagulation diameter is 8 to $10 \mathrm{~mm}$. Varadarajulu et al. (11) utilized EUS-RFA with a 19-gauge fine needle aspiration (FNA) needle fitted with an umbrella-shaped, retractable needle electrode array and they achieved a complete coagulation necrosis of $2.6 \mathrm{~cm}$ in diameter in the liver of 5 Yucatan pigs without damage to the surrounding parenchyma or vasculature.

We aimed at applying multiple needle insertions and low ablation power to achieve complete coagulation necrosis while still minimizing the risk of damage to the intestinal mucosa. Hence, here we showed our preliminary result of conducting multiple-round EUS-RFA with low ablation power for unresectable pancreatic cancer and evaluated the technical feasibility, safety and efficacy. We present the following article in accordance with the STROBE reporting checklist (available at http://dx.doi.org/10.21037/apm-201468).

\section{Methods}

\section{Patients}

In the present study, data of eleven patients with unresectable pancreatic cancer referred to EUS-RFA were prospectively collected from Tongji Hospital of Tongji Medical College of Huazhong University of Science and Technology between November 2013 and November 2018, and retrospectively analysed. All of the patients were diagnosed as pancreatic cancer by FNA cytology and gave written informed consent before the EUS-RFA procedure. The study was approved by the local Ethics Committees of Tongji Hospital of Tongji Medical College of Huazhong University of Science and Technology (TJ-IRB20190611) and conformed to the provisions of the Declaration of Helsinki (as revised in 2013).

\section{EUS-RFA procedure}

RFA was performed under deep sedation according to the principles of "monitored anaesthesia care". The patients were anaesthetized with intravenous administration of propofol. All patients received oxygen during the procedures; blood pressure and heart rate were monitored.

The procedure was performed by a single experienced endosonographer with more than 5 years of EUS experience, who had performed more than 300 EUS procedures and more than 150 FNA annually. The Olympus linear echoendoscope (GF-UCT 260; Olympus, Tokyo, Japan) platform on an ultrasound processor was manoeuvred to obtain proper sonographic visualization of the target lesion. The ultrasound processors we used including Alpha 5 (Aloka, Tokyo, Japan), SU-8000 (Fujifilm, Tokyo, Japan), EU-ME1 (Olympus, Tokyo, Japan), and EU-ME2 (Olympus, Tokyo, Japan). The Habib ${ }^{\mathrm{TM}}$ EUS RFA (EMcision Ltd., London, $\mathrm{UK})$ is a 1 Fr wire $(0.33 \mathrm{~mm}, 0.013$ ”), which has a working length of $190 \mathrm{~cm}$, that can be inserted through the biopsy 
channel of an echoendoscope, and the length of the active tip is $2 \mathrm{~cm}$. RFA power is applied to the active tip to coagulate tissue in the pancreas. Under EUS control, a 22-gauge FNA needle (with stylet) (Echo Tip; Cook Endoscopy, Ireland) was introduced into the target lesion. The tip of FNA needle was then positioned at the deepest part of the tumour through the gastric wall or duodenum. The stylet was removed from the FNA needle and the Habib ${ }^{\mathrm{TM}} \mathrm{EUS}$ RFA catheter was gently pushed inside the hollow of the FNA needle until it could not be pushed farther. Carefully maintaining this position of the probe, the FNA needle was gradually withdrawn by $2-2.5 \mathrm{~cm}$ to disengage contact between the active part of the RF catheter located at the tip and the metallic FNA needle (12).

After insertion of the RFA electrode into the mass, the radiofrequency generator (RITA System Generator 1500X; RITA Medical Systems, California, USA) was applied. Generator power was set to 5-10 watts. The ablation was performed for 90 seconds per site, and was repeated until the hyperechoic zone around the electrode tip sufficiently covered the tumour.

\section{Follow-up}

Patients were kept in hospital at least for 2 days to evaluate the safety of the RFA treatment (clinical symptoms and laboratory tests including complete blood count, liver function tests, serum amylase/lipase levels). Serum CA199, computed tomography (CT) and magnetic resonance imaging (MRI) were performed before the procedure and at 1 month after the procedure. Clinical follow-up (consisting of CA19-9, imaging examination, clinical signs and survival time) was conducted at each other month after the procedure.

\section{Statistical analysis}

Categorical variables were described in terms of the numbers or percentages, whereas continuous variables were described as the mean (range). All of the statistical analyses were performed with SAS version 9.2 (SAS Institute Inc, Cary, NC, USA).

\section{Results}

\section{Patients}

Eleven patients with a median age of 64.7 (range, 42-83) years,
5 women and 6 men, were evaluated in this study. The patients presented to our hospital with common symptoms, including abdominal pain, jaundice, and diarrhoea. Diagnosis of pancreatic cancer was based on imaging reviewed by an expert radiologist, and was further confirmed by diagnostic FNA cytology. All patients were deemed not suitable for surgical intervention. Locally advanced disease was present in 7 patients, and 4 patients had metastasis. The pancreatic cancer was located in the head of the pancreas in four cases, in the pancreas neck in three cases, in the pancreas body in three case, and in the tail of the pancreas in one case. Table 1 provides the patient characteristics and procedure specifications.

\section{Feasibility}

The mean diameter of the tumours was $28.0 \mathrm{~mm}$. In larger lesions, the Habib ${ }^{\mathrm{TM}}$ EUS RFA probe and needle was pulled back as one unit and repositioned to ablate near the proximal end of the lesion. This process could be repeated as many times as needed, to ensure complete ablation of the lesion. In case 1 and case 2, RFA was applied at 10 watts three times, respectively. In the other nine cases, RFA was applied at 5 watts four times, three times or two times according to the lesion size, and the same procedure was repeated 1 week later. In case 3 , in which the lesion size was $29.7 \mathrm{~mm}, \mathrm{RFA}$ was applied at 5 watts twice in one session, and the same procedure was repeated every other week for 8 total sessions. Table 2 summarizes the key outcomes.

Technical success was judged as the correct placement of the needle in the lesion to generate an efficient ablation wave and hyperechoic zone during the procedure (Figure 1). In this context, EUS-RFA was performed successfully in all cases.

\section{Safety}

There were no procedure-related deaths and no major complications observed post-procedure, including pancreatitis, bleeding, duodenal injury, or portal vein and/or splenic vein thrombosis (13). Only two patient (Case 5 and Case 9) experienced mild abdominal pain without detectable pancreatic enzyme elevation, and the condition improved in 24 hours.

\section{Efficacy}

Technical efficacy was evaluated by tumour size, serum 
Table 1 Patient characteristics and procedure specifications

\begin{tabular}{llllllll}
\hline No. & Age & Sex & $\begin{array}{c}\text { Lesion } \\
\text { location }\end{array}$ & Metastasis & Chemotherapy & $\begin{array}{c}\text { Power } \\
\text { (watts) }\end{array}$ & $\begin{array}{c}\text { No. of RF } \\
\text { applications/session }\end{array}$ \\
\hline Case 1 & 62 & Woman & Head & No & Yes & 10 & 3 \\
of sessions
\end{tabular}

No., number; RF, radiofrequency.

Table 2 Outcomes after EUS-RFA of pancreatic cancer

\begin{tabular}{|c|c|c|c|c|c|c|c|}
\hline No. & $\begin{array}{l}\text { Pre ablation } \\
\text { size* }^{\star}(\mathrm{mm})\end{array}$ & $\begin{array}{c}\text { Post ablation } \\
\text { size }^{\star}(\mathrm{mm})\end{array}$ & $\begin{array}{l}\text { Pre CA19-9 } \\
\text { (U/mL) }\end{array}$ & $\begin{array}{l}\text { Post CA19-9 } \\
(\mathrm{U} / \mathrm{mL})\end{array}$ & Complications & $\begin{array}{c}\text { Duration of } \\
\text { follow-up in months }\end{array}$ & Results of follow-up \\
\hline Case 1 & $32.2 \times 29.4$ & No change & $>1,200$ & 570 & No & 9 & Died 9 months after treatment \\
\hline Case 3 & $29.7 \times 17.2$ & $26.5 \times 11.7$ & 69.8 & 57.5 & No & 12 & Alive \\
\hline Case 6 & $19.4 \times 17.6$ & No change & 45.7 & 44.3 & No & 5 & Died 5 months after treatment \\
\hline Case 7 & $28.0 \times 24.0$ & No change & 77.5 & 64.0 & No & 4 & Died 4 months after treatment \\
\hline Case 8 & $33.0 \times 27.0$ & No change & 558.3 & 236.4 & No & 4 & Died 4 months after treatment \\
\hline Case 10 & $16.4 \times 15.6$ & No change & $<0.60$ & / & No & 5 & Died 5 months after treatment \\
\hline Case 11 & $38.0 \times 25.0$ & No change & 39.6 & 35.4 & No & 4 & Died 4 months after treatment \\
\hline
\end{tabular}

*, the size was measured on computed tomography (CT). EUS-RFA, endoscopic ultrasound-guided radiofrequency ablation; No., number.

CA19-9, tumour apparent diffusion coefficient (ADC) value of MRI-diffusion-weighted imaging (DWI) and ablated area percentage measurement one month after the procedure. In 2 patients (case 3 and case 5) the tumour size decreased, and the serum CA19-9 levels decreased in 5 patients (case 1 , case 4 , case 5 , case 8 and case 9) (Table 2). In one patient (case 3), we observed increased tumour ADC value (Figure 2) and 20\% ablated area inside the tumour (Figure 3), indicating a favourable response to cancer therapy (14).

Case 3, a 62-year-old woman had locally advanced pancreatic cancer confirmed by EUS-FNA cytology. She underwent eight sessions of RFA every other week and 

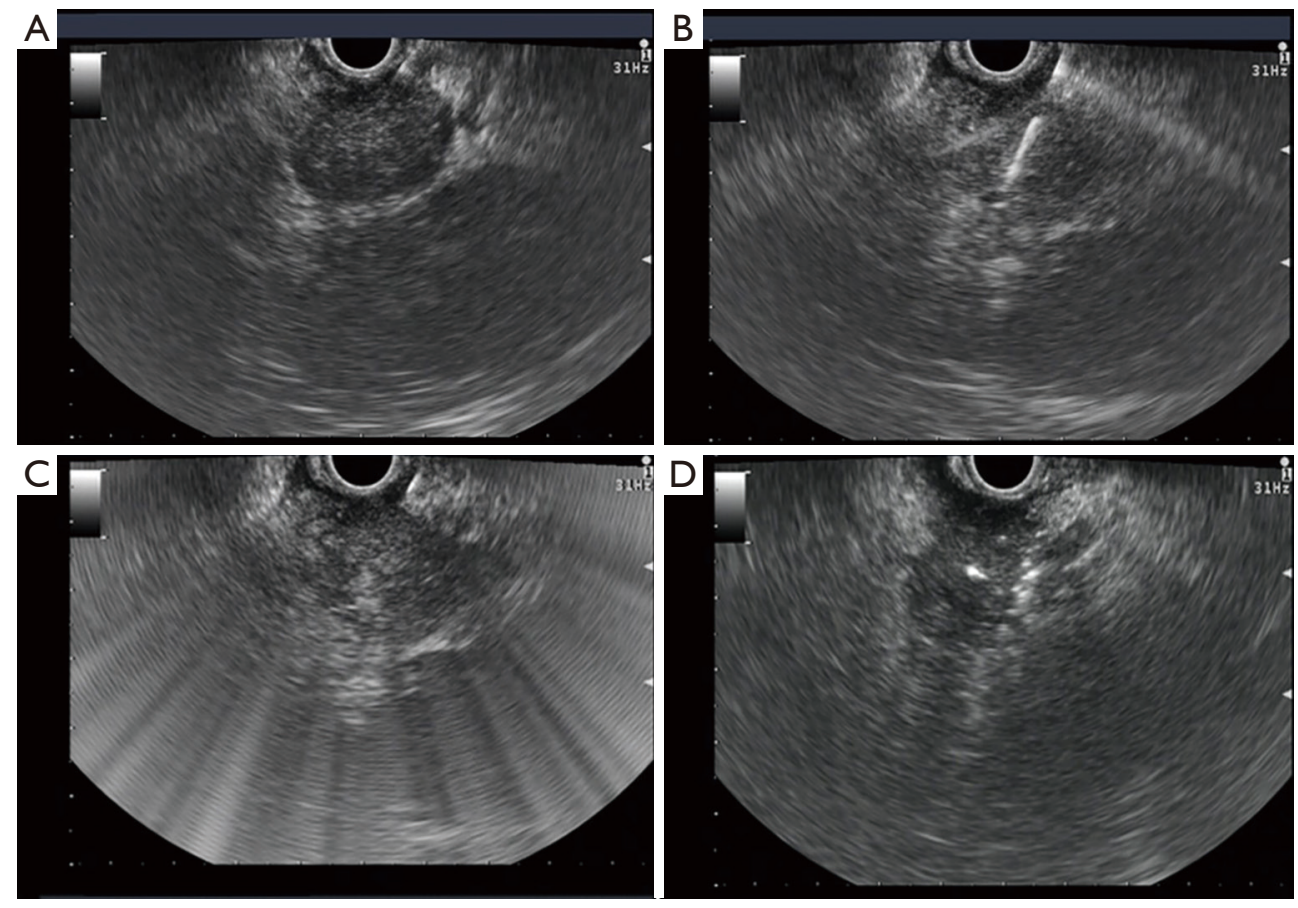

Figure 1 Endoscopic ultrasonography (EUS)-radiofrequency ablation (RFA) procedure. (A) EUS images of the pancreatic mass; (B) fine needle aspirations were performed; (C) the ablation was performed, and an ablation wave was observed; (D) a hyperechoic zone around the electrode tip was observed.

tolerated the treatment well. After the third session of RFA, we observed ablated area $(12.0 \mathrm{~mm} \times 8.5 \mathrm{~mm})$ by EUS (Figure 3), and the patient had no signs of necrotizing pancreatitis. One month after the procedure was finished, CT imaging showed a reduced tumour size and MRI imaging show the increased tumour ADC (Figure 2). The patient was then followed for twelve months and at the time drafting this article, she remained alive without pain or jaundice.

\section{Discussion}

Under EUS guidance, RFA energy can target pancreatic lesions accurately. The use of RFA has expanded to treat more pancreatic diseases, including pancreatic cystic neoplasms and neuroendocrine tumours, as well as pancreatic cancer (15-17).

Recent study involving 10 adult mini pigs, Kim et al. (10) demonstrated safety, feasibility, and efficacy for EUS-RFA of the pancreatic body and tail. The pathology results also confirmed a well-demarcated spherical focus of coagulation necrosis in RFA sites. In a study of 5 Yucatan pigs, Gaidhane et al. (18) further demonstrated that EUS-RFA of the pancreatic head was well tolerated with minimal pancreatitis. Barthet et al. (15) reported that EUS-RFA performed on 29 patients with pancreatic neuroendocrine tumours or cystic neoplasms was safe with a $10 \%$ complication rate, which can be decreased by improved prophylaxis for the procedure. Song et al. (13) applied EUS-RFA in 6 patients with unresectable pancreatic cancer (4 patients had locally advanced disease and 2 had metastasis). In their study, only 2 patients experienced mild abdominal pain, with no other adverse events. In addition, they found that the necrotic areas induced by RFA tended to increase blood flow on contrast-enhanced EUS, which might indicate the potential of RFA to enhance the efficacy of systemic chemotherapy.

Though EUS-RFA is technically successful in many cases, there have been clinical complications associated with the technique, including acute pancreatitis, pancreatic leaks, infection of necrotic pancreatic tissue, and posttreatment bleeding. Another problem is to achieve complete coagulation necrosis of pancreatic lesions with large diameter. It thus underlines the importance of choosing appropriate time and power used during ablation. As suggested by others, using lower energy may allow for 

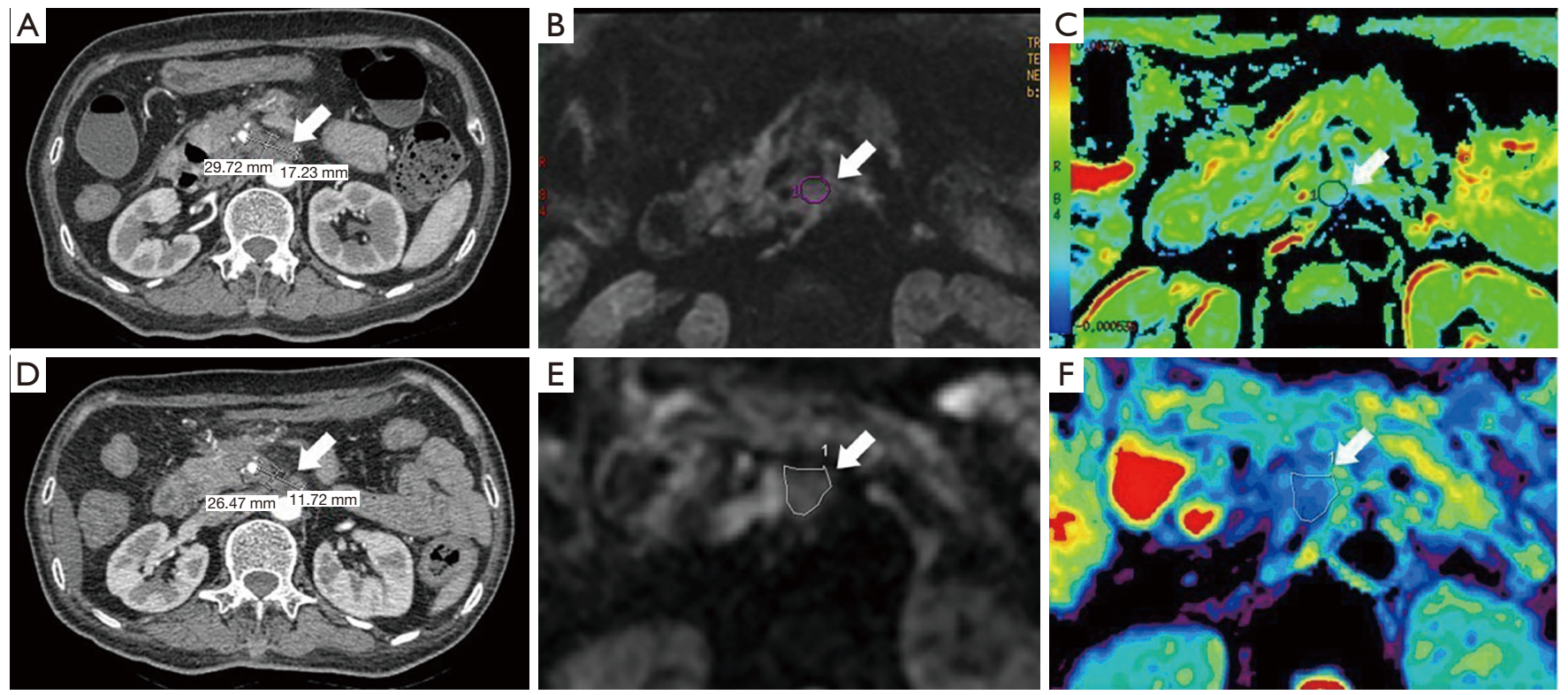

Figure 2 Images of case 3 indicate the change of the tumour after Endoscopic ultrasound (EUS)-radiofrequency ablation (RFA). (A) Computed tomography (CT) image of the pancreatic mass, which measured $29.7 \times 17.2 \mathrm{~mm}$ prior to EUS-RFA; (B) magnetic resonance imaging diffusion-weighted imaging (MRI-DWI) (b-value 1,000 s/ $\mathrm{mm}^{2}$ ) shows the ROI (region of interest, arrow) before therapy; (C) colorcoded apparent diffusion coefficient (ADC) map of the pancreatic mass before therapy, and the tumour $\mathrm{ADC}$ is $997.0 \times 10^{-6} \mathrm{~mm} / \mathrm{s}$ in the $\mathrm{ROI}$ (arrow); (D) a CT scan performed 1 month after EUS-RFA, showing that the size of pancreatic mass was $26.5 \times 11.7 \mathrm{~mm}$; (E) MRI-DWI (b-value 1,000 s/ $\mathrm{mm}^{2}$ ) shows the ROI (arrow) after therapy; (F) color-coded ADC map of the pancreatic mass after therapy, and the tumour ADC is $1,397.3 \times 10^{-6} \mathrm{~mm}^{2} / \mathrm{s}$ in the ROI (arrow).
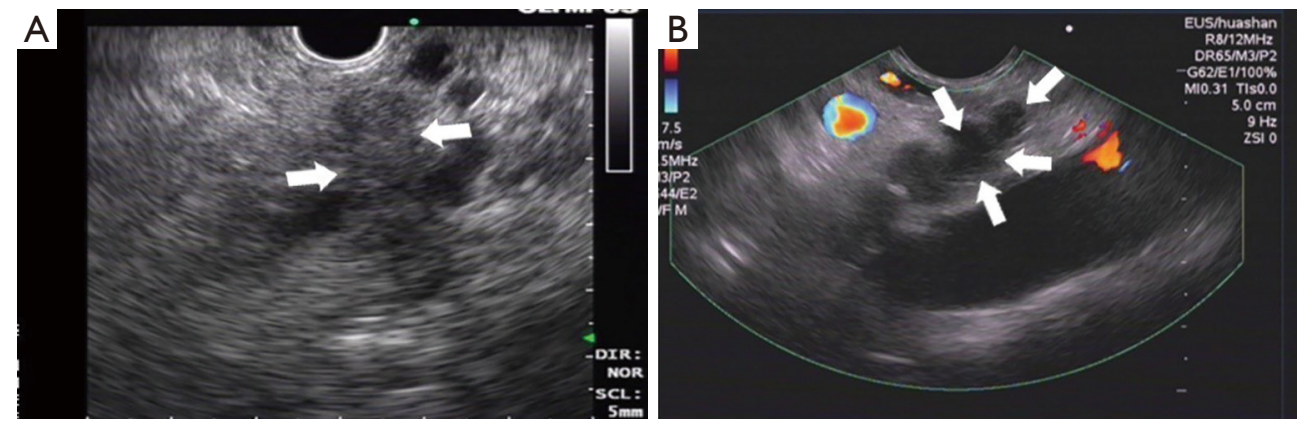

Figure 3 Endoscopic ultrasound (EUS) images show the coagulative necrosis generated after radiofrequency ablation (RFA). (A) EUS images of the pancreatic mass (arrow) before EUS-RFA; (B) EUS images of the ablated area (arrow) after three sessions of EUS-RFA.

multiple ablations with lower morbidity and two or more procedures may be necessary in many cases $(11,18)$.

Here we showed our preliminary study of conducting multiple-round EUS-RFA with low power for unresectable pancreatic cancer. In our first two patients, we observed charring of the pancreatic tissue that tended to interfere with thermal conduction. Therefore, we reduced power for the remainder of study. Yoon et al. (19) used the Habib ${ }^{\mathrm{TM}}$ monopolar EUS RFA electrode in their porcine pancreas model. The mean maximum diameters of necrotic areas generated by $3,4,5,10,15$, and 25 watts energy were $2.5 \pm 0.7,4.0 \pm 1.4,8.0 \pm 1.7,6.3 \pm 1.5,4.7 \pm 1.2$, and $4.5 \pm$ $0.7 \mathrm{~mm}$, respectively. It seems that using 5 watts is sufficient to achieve the maximum coagulative necrosis $(8.0 \pm$ $1.7 \mathrm{~mm}$ ). We carefully chose 5 watts for case 3 and observed a discrete zone of coagulation necrosis during follow-up. 
In current study, we used the RFA electrode transmitting monopolar RF energy through the RITA System Generator 1500X. Accordingly, the tips of the RITA hooks have thermocouples that report real-time temperatures at the treatment volume margin, as the tissue heats up, which automatically maximizes treatment volume. Thus, the RFA electrode provides linearly, scalable ablations up to $2 \mathrm{~cm}$ in length and $1 \mathrm{~cm}$ in diameter. For case 3 , the tumour was $29.7 \times 17.2 \mathrm{~mm}$. Ideally, a maximum of 2 to 3 needle insertions could be made into the lesion for each ablation session. For safety and efficiency purposes, RFA was conducted with 5 watts ablation power twice in one session, and the same procedure was repeated every other week for 8 total sessions. After the third session of RFA, we observed that the maximal coagulative necrosis was $12 \times 8.5 \mathrm{~mm}$ by EUS (Figure 3). In case 4 to 11, RFA was applied at 5 watts four times, three times or two times according to the lesion size. Nevertheless, the same procedure was only repeated once, 1 week later, mainly because the patients could not afford the EUS-RFA cost.

We evaluated main outcomes, including technical success and short-term efficacy (tumour response), measured by the tumour size, the tumour ADC, CA199 level and ablated area percentage. MRI-DWI technique depicts molecular diffusion and has been used for the depiction and characterization of tumours in oncological imaging (20). DWI is valued by ADC. A significant reduction in the diffusion properties of water protons in solid tumour and the resulting reduction in the measured ADC value of tumour relative to normal tissue have been well documented and now is often used to predict the early response of solid tumours to an effective therapy $(14,21)$. Thus, the tendency for ADC to decrease as tumour volume increases whereas the increasing ADC reflects the favourable response to cancer therapy (14).

We observed case 3 who survived one year after followup and showed increased ADC value of DWI and 20\% ablated area, indicating survival benefit maybe achieved. It has been reported that the decrease of CA 19-9 during chemotherapy with gemcitabine predicts survival time in patients with advanced pancreatic cancer (22). However, cases in our study such as case 4 and case 5 who were found died due to metastasis although had reduced CA19-9 value after procedure, it seems that EUS-RFA did not provide a survival benefit. In the future, a case series study is required to confirm our results.

The obvious drawback of this ablative method is its palliative nature, given the possibility of growth of the remnant tumour (23). However, recent bench research suggested that RFA-mediated adaptive immunity also plays important anticancer roles by recruiting immune cells to ablation sites and subsequently releasing antitumour responses $(24,25)$. Dromi et al. (26) reported that RFA treatment results in enhanced anti-tumour T-cell immune responses. Another study suggested that RFA might stimulate antigen specific anti-tumour immunity by inducing expression of heat shock protein 70 (27). Given that RFA generates large amounts of cellular debris and stimulates necrotic cell death which may activate local dendritic cells, our method using multiple-round EUSRFA with low ablation power may continuously create a proimmune environment both locally and systemically and therefore contribute to the enhanced adaptive immunity.

Our study presents the following limitations: a small cohort of patients, a short follow-up period, and their disease was heterogeneous. Moreover, given the short-time interval in analysis and the discontinuing after two round RFA treatments in most patients, it is still not possible to deduce any conclusion regarding the improvement in the survival rate after RFA procedure.

\section{Conclusions}

The present case series showed that a multiple-round ablation with optimal RF energy is feasible, safe, and shortterm efficacy for patients with locally advanced pancreatic cancer. However, the long-term efficacy of this novel treatment method should be further assessed in properly designed studies.

\section{Acknowledgments}

Thanks are due to all the staff in in the Digestive Endoscopy Center of our hospital for providing the clinical data.

Funding: This work was supported in part by grants of the National Natural Science Foundation of China (81802418, 81802427).

\section{Footnote}

Reporting Checklist: The authors have completed the STROBE Statement Checklist. Available at http://dx.doi. org/10.21037/apm-20-1468

Data Sharing Statement: Available at http://dx.doi. org/10.21037/apm-20-1468 
Peer Review File: Available at http://dx.doi.org/10.21037/ apm-20-1468

Conflicts of Interest: All authors have completed the ICMJE uniform disclosure form (available at http://dx.doi. org/10.21037/apm-20-1468). The authors have no conflicts of interest to declare.

Ethical Statement: The authors are accountable for all aspects of the work in ensuring that questions related to the accuracy or integrity of any part of the work are appropriately investigated and resolved. The trial was conducted in accordance with the Declaration of Helsinki (as revised in 2013). The study was approved by the local Ethics Committees of Tongji Hospital of Tongji Medical College of Huazhong University of Science and Technology (TJ-IRB20190611) and informed consent was taken from all the patients.

Open Access Statement: This is an Open Access article distributed in accordance with the Creative Commons Attribution-NonCommercial-NoDerivs 4.0 International License (CC BY-NC-ND 4.0), which permits the noncommercial replication and distribution of the article with the strict proviso that no changes or edits are made and the original work is properly cited (including links to both the formal publication through the relevant DOI and the license). See: https://creativecommons.org/licenses/by-nc-nd/4.0/.

\section{References}

1. Siegel RL, Miller KD, Jemal A. Cancer statistics, 2020. CA Cancer J Clin 2020;70:7-30.

2. Sohal DPS. Adjuvant and neoadjuvant therapy for resectable pancreatic adenocarcinoma. Chin Clin Oncol 2017;6:26.

3. Tabernero J, Macarulla T. Changing the paradigm in conducting randomized clinical studies in advanced pancreatic cancer: an opportunity for better clinical development. J Clin Oncol 2009;27:5487-91.

4. Philip PA, Mooney M, Jaffe D, et al. Consensus report of the national cancer institute clinical trials planning meeting on pancreas cancer treatment. J Clin Oncol 2009;27:5660-9.

5. Toyoda M, Kakizaki S, Horiuchi K, et al. Computed tomography-guided transpulmonary radiofrequency ablation for hepatocellular carcinoma located in hepatic dome. World J Gastroenterol 2006;12:608-11.
6. Chen MH, Yang W, Yan K, et al. Treatment efficacy of radiofrequency ablation of 338 patients with hepatic malignant tumor and the relevant complications. World J Gastroenterol 2005;11:6395-401.

7. Girelli R, Frigerio I, Salvia R, et al. Feasibility and safety of radiofrequency ablation for locally advanced pancreatic cancer. Br J Surg 2010;97:220-5.

8. Casadei R, Ricci C, Pezzilli R, et al. A prospective study on radiofrequency ablation locally advanced pancreatic cancer. Hepatobiliary Pancreat Dis Int 2010;9:306-11.

9. Matthes K, Mino-Kenudson M, Sahani DV, et al. Concentration-dependent ablation of pancreatic tissue by EUS-guided ethanol injection. Gastrointest Endosc 2007;65:272-7.

10. Kim HJ, Seo DW, Hassanuddin A, et al. EUS-guided radiofrequency ablation of the porcine pancreas. Gastrointest Endosc 2012;76:1039-43.

11. Varadarajulu S, Jhala NC, Drelichman ER. EUS-guided radiofrequency ablation with a prototype electrode array system in an animal model (with video). Gastrointest Endosc 2009;70:372-6.

12. Pai M, Habib N, Senturk H, et al. Endoscopic ultrasound guided radiofrequency ablation, for pancreatic cystic neoplasms and neuroendocrine tumors. World J Gastrointest Surg 2015;7:52-9.

13. Song TJ, Seo DW, Lakhtakia S, et al. Initial experience of EUS-guided radiofrequency ablation of unresectable pancreatic cancer. Gastrointest Endosc 2016;83:440-3.

14. Kim H, Keene KS, Sarver DB, et al. Quantitative perfusion- and diffusion-weighted magnetic resonance imaging of gastrointestinal cancers treated with multikinase inhibitors: a pilot study. Gastrointest Cancer Res 2014;7:75-81.

15. Barthet M, Giovannini M, Lesavre N, et al. Endoscopic ultrasound-guided radiofrequency ablation for pancreatic neuroendocrine tumors and pancreatic cystic neoplasms: a prospective multicenter study. Endoscopy 2019;51:836-42.

16. Scopelliti F, Pea A, Conigliaro R, et al. Technique, safety, and feasibility of EUS-guided radiofrequency ablation in unresectable pancreatic cancer. Surg Endosc 2018;32:4022-8.

17. Crino SF, D'Onofrio M, Bernardoni L, et al. EUS-guided Radiofrequency Ablation (EUS-RFA) of Solid Pancreatic Neoplasm Using an 18-gauge Needle Electrode: Feasibility, Safety, and Technical Success. J Gastrointestin Liver Dis 2018;27:67-72.

18. Gaidhane M, Smith I, Ellen K, et al. Endoscopic Ultrasound-Guided Radiofrequency Ablation (EUS-RFA) 
of the Pancreas in a Porcine Model. Gastroenterol Res Pract 2012;2012:431451.

19. Yoon WJ, Daglilar ES, Kamionek M, et al. Evaluation of radiofrequency ablation using the 1-Fr wire electrode in the porcine pancreas, liver, gallbladder, spleen, kidney, stomach, and lymph nodes: A pilot study. Dig Endosc 2015. [Epub ahead of print]. doi: 10.1111/den.12575.

20. Stecco A, Buemi F, Cassara A, et al. Comparison of retrospective PET and MRI-DWI (PET/MRI-DWI) image fusion with PET/CT and MRI-DWI in detection of cervical and endometrial cancer lymph node metastases. Radiol Med 2016;121:537-45.

21. Tsien C, Cao Y, Chenevert T. Clinical applications for diffusion magnetic resonance imaging in radiotherapy. Semin Radiat Oncol 2014;24:218-26.

22. Halm U, Schumann T, Schiefke I, et al. Decrease of CA 19-9 during chemotherapy with gemcitabine predicts survival time in patients with advanced pancreatic cancer. Br J Cancer 2000;82:1013-6.

Cite this article as: Wang J, Wang Y, Zhao Y, Wu X, Zhang M, Hou W, Chen Q, Cheng B. Endoscopic ultrasound-guided radiofrequency ablation of unresectable pancreatic cancer with low ablation power and multiple applications: a preliminary study of 11 patients. Ann Palliat Med 2021;10(2):1842-1850. doi: 10.21037/apm-20-1468
23. Lakhtakia S, Ramchandani M, Galasso D, et al. EUSguided radiofrequency ablation for management of pancreatic insulinoma by using a novel needle electrode (with videos). Gastrointest Endosc 2016;83:234-9.

24. McGahan JP, Brock JM, Tesluk H, et al. Hepatic ablation with use of radio-frequency electrocautery in the animal model. J Vasc Interv Radiol 1992;3:291-7.

25. Bartholomae WC, Rininsland FH, Eisenberg JC, et al. T cell immunity induced by live, necrotic, and apoptotic tumor cells. J Immunol 2004;173:1012-22.

26. Dromi SA, Walsh MP, Herby S, et al. Radiofrequency ablation induces antigen-presenting cell infiltration and amplification of weak tumor-induced immunity. Radiology 2009;251:58-66.

27. Teng LS, Jin KT, Han N, et al. Radiofrequency ablation, heat shock protein 70 and potential anti-tumor immunity in hepatic and pancreatic cancers: a minireview. Hepatobiliary Pancreat Dis Int 2010;9:361-5. 